\title{
La psiconefrología: un campo de estudio en desarrollo
}

\section{Psychonephrology: A field of study in development}

\author{
Alejandra García-Arista ${ }^{1}$ y Aranel de Jesús Arredondo-Pantaleón ${ }^{2}$
}

\begin{abstract}
RESUMEN
El objetivo de este artículo fue describir los antecedentes y la situación actual de la psicología en el campo de la nefrología y la necesidad de consolidarla como una especialidad. Se detallan asimismo sus aportaciones en los ambientes hospitalarios y de la salud. Se discute su utilidad e impacto en la mejora de la calidad de vida del paciente con enfermedad renal crónica, señalando las áreas de acción del psiconefrólogo y las técnicas de tratamiento cognitivo-conductual más utilizadas en dicho contexto de intervención. Finalmente, se identifican algunos de los desafíos que la psiconefrología enfrenta en su integración al equipo interdisciplinario de atención a los pacientes con dicha enfermedad.
\end{abstract}

Palabras clave: Psicología de la salud; Intervención psicológica; Enfermedad renal crónica; Nefrología.

\begin{abstract}
The aim of this paper was to describe the background and current situation of psychology in the field of nephrology. It addresses the need for the consolidation of psychonephrology as a specialty. Contributions in the hospital and health environment are also emphasized. We also discuss its utility and impact on improving quality of life of patients with chronic kidney disease. The areas of action of the psychonephrologist are examined and analyses show that cognitive behavioral treatments are the most frequently used in this intervention context. Finally, we identify some of the challenges that psychonephrology faces to be integrated into the interdisciplinary team of patients with chronic kidney disease.
\end{abstract}

Key words: Health psychology; Psychological intervention; Chronic kidney disease; Nephrology.

$\mathrm{E}$ n los últimos años, la psicología ha ampliado su campo de aplicación y especialización en concordancia con los requerimientos de la población a la que están orientados su estudio e intervención. La psicología comparte modelos, teorías y técnicas con otras disciplinas y fortalece a las nacientes. Tal es el caso de la psicología de la salud, disciplina que nació en la década de los 70 al surgir nuevas y urgentes necesidades de atención primaria (Oblitas y Becoña, 2000), beneficiando así a una cantidad considerable de personas al disminuir su sufrimiento y favorecer en ellas una mejor calidad de vida (Bazán, 2003). Un hito relevante que influyó en el desarrollo de esta área fue la definición de salud establecida por la World Health Organization (WHO) (1946), misma que reconoce claramente la influencia de factores psicosociales en la salud física, premisa que sostienen diversos autores al definir la psicología de la salud (Amigo, 2014; Piña y Rivera, 2006).

\footnotetext{
${ }^{1}$ Departamento de Ciencias de la Salud, Universidad Autónoma Metropolitana, Unidad Lerma, Av. Hidalgo 46, Col. La Estación, 52006 Lerma de Villada, Edo. de México, México, tel. (728)282-70-02, ext.2014, correo electrónico: a.garcia@correo.ler.uam.mx. Artículo recibido el 29 de septiembre y aceptado el 23 de noviembre de 2017.

${ }^{2}$ Programa de Doctorado en Psicología y Salud, Facultad de Psicología. Universidad Nacional Autónoma de México, Av. Universidad 3114, Col. Copilco Universidad, Del. Coyoacán, 04510 Ciudad de México, México, correo electrónico: aranel_arredondo_pantaleon@yahoo.com.

Citación: García-Arista, A. y Arredondo-Pantaleón, A.J. (2018). La psiconefrología: un campo de estudio en desarrollo. Psicología y Salud, 28(2), 261-269.
} 
De esta manera, la psicología se encargó de destinar una parte importante de sus contribuciones educativas, científicas y profesionales a la promoción y mantenimiento de la salud; la prevención y el tratamiento de enfermedades; la identificación de etiologías, diagnósticos y comorbilidades, así como el mejoramiento del sistema de cuidados de la salud y la conformación de políticas públicas (Weiner, 1982).

Con estos antecedentes, la psicología se ha ido abriendo camino para ganar un lugar en la atención integral de las enfermedades, pues entre sus objetivos para el mantenimiento de la salud se encuentra la modificación de variables conductuales, emocionales y cognitivas. A partir de estos alcances, se ha ido especializando cada vez más en respuesta al incremento masivo de padecimientos crónicos no transmisibles. Un ejemplo de ello es la psicooncología, disciplina especializada en el estudio e intervención de pacientes oncológicos y sus familias, así como del personal sanitario que los atiende (Ascencio, 2015). Al igual que el cáncer, existen otras enfermedades crónicas que han mostrado un aumento sustancial durante los últimos años, las que, además de generar un deterioro físico, producen malestar emocional significativo y afectan considerablemente la esfera social del paciente y su familia (Piña e Ybarra, 2015).

Una de las enfermedades crónico-degenerativas cuya población afectada va en aumento es la enfermedad renal crónica (ERC en lo sucesivo), definida como la disminución de la capacidad funcional de los riñones de forma permanente e irreversible (Marques y Barrientos, 2000), que ocasiona que estos pierdan su capacidad para eliminar los productos de desecho, mantener en equilibrio la cantidad de líquidos en el organismo, controlar la presión arterial, facilitar la producción y mantenimiento de los glóbulos rojos y regular los niveles de calcio y fósforo (Pérez, Llamas y Legido, 2005).

La ERC es un padecimiento multifactorial cuyas causas más comunes son la glomerulonefritis, la poliquistosis renal, las nefropatías intersticiales, las anomalías congénitas, las enfermedades autoinmunes y sistémicas, la enfermedad hipertensiva y la diabetes mellitus tipo II (Hersh-Rifkin y Stoner, 2005; Marques y Barrientos, 2000). En México, esta última es la responsable de $48.5 \%$ de los ca- sos de ERC, lo que la convierte en la principal causa de este problema de salud (Ávila, Conchillos, Rojas, Ordoñez y Ramírez, 2013; Méndez, Méndez, Tapia, Muñoz y Aguilar, 2010). En España, se calcula que $35 \%$ de los pacientes con diabetes mellitus tipo II sufren afectaciones renales en algún grado (Martínez, Navarro y Górriz, 2012).

El tratamiento de la ERC no logra una recuperación total de la salud del paciente (Andrés, 2004), por lo que se considera conservador, ya que su objetivo principal es mantener estable a la persona y prevenir complicaciones (National Kidney Foundation, 2002). Incluye procedimientos sumamente invasivos y demandantes, lo que implica un elevado costo físico, psicológico y económico para el paciente y su familia.

El manejo médico de esta enfermedad incluye cuatro aspectos básicos: el tratamiento sustitutivo de la función renal (diálisis peritoneal, hemodiálisis o trasplante), el componente farmacológico, una nutrición ajustada a la situación renal y la restricción de líquidos (García, Fajardo, Guevara, González y Hurtado, 2002; Páez, Jofré, Azpiroz y Bortoli, 2009).

\section{Implicaciones psicológicas de la enfermedad renal crónica}

Además de los problemas físicos, los pacientes con ERC se encuentran expuestos a diversas fuentes de estrés psicológico, como la dependencia al tratamiento sustitutivo, el requisito de respuesta implicado en el apego terapéutico, las pérdidas sufridas como consecuencia del estado de salud (trabajo, libertad, tiempo de esparcimiento, esperanza de vida, etc.), la disfunción sexual asociada (Muñoz et al., 2010) y la incertidumbre respecto al futuro, lo que en conjunto genera sentimientos de minusvalía, incapacidad e indefensión (Vázquez, Vital, Bravo, Jurado y Reynoso, 2009), pensamientos y conductas de dependencia, y respuestas emocionales como ira, hostilidad y estrés (Rivera, Montero y Sandoval, 2012). De igual forma, quienes la padecen deben realizar múltiples ajustes físicos y psicológicos en las áreas laboral, económica, social y familiar para adaptarse al nuevo estilo de vida y a los cambios que conlleva la enfermedad.

Se ha visto que las alteraciones psicológicas que mayormente se presentan en personas con ERC 
son depresión (Aggarwal, Jain, Dabas y Yadav, 2017; Ćwiek et al., 2017; Rojas, Ruiz y González, 2017), ansiedad (Cabrales, Sánchez, Ojeda, Monterrubio y Hernández, 2017; Contreras, Esguerra, Espinoza, Gutiérrez y Fajardo, 2006; García y Calvanese, 2008; Lerma, 2012), alteraciones del sueño (Iliescu, Yeates y Holland, 2004; Liaveri et al., 2017), desesperanza (Cabrales et al., 2017), procesos fóbico-obsesivos y rituales, y deterioro de la autoimagen (María, 2003), lo que en conjunto, repercute negativamente en la percepción del paciente acerca de su calidad de vida (Calvanese, Feldman y Weisinger, 2004).

La escasa adherencia al tratamiento constituye un problema ampliamente extendido en los pacientes con ERC (Amigo, 2014; Conde, 1994; Cvengros, Christensen y Lawton, 2004). Dicha adherencia es un predictor central del establecimiento, curso y pronóstico del padecimiento (Santillán, 2010). El incumplimiento de las indicaciones del personal de salud puede generar consecuencias tales como malnutrición, neuropatías, enfermedades óseas, falla cardiaca e incluso la muerte (Andrés, 2004). De acuerdo con la Organización Mundial de la Salud (2004), la escasa adherencia al régimen terapéutico compromete de manera importante su efectividad, disminuye la percepción de bienestar de los pacientes y puede generar costos extras al sistema sanitario, por lo que propone que las intervenciones deben ir encaminadas a mejorar esta situación y a hacer una aportación importante a la prevención secundaria (modificación de conductas) y terciaria (disminuir las consecuencias de la enfermedad) en los pacientes con ERC.

El paciente se encuentra regularmente inmerso en un sistema familiar, por lo que los retos y desafíos a los que se enfrenta no son exclusivos de quien padece la enfermedad, pues este trastorno demanda un ajuste físico, psicológico, social y ambiental, al igual que una reasignación de roles por parte de la familia en función de las necesidades del paciente, quien requiere tareas de asistencia y apoyo complejas, mismas que generalmente recaen en un miembro del sistema familiar, al que se conoce como cuidador primario informal, que es la persona que desempeña las labores de cuidado y acompañamiento, que no se encuentra adscrita a una institución de salud y que no recibe remuneración alguna por el desempeño de esas tareas (Montero, 2013).

El cuidador modifica sus rutinas y su estilo de vida; reduce sus horas laborales retribuidas y las de esparcimiento, además de que frecuentemente descuida su propia salud para desempeñar óptimamente sus funciones; se enfrenta a estresores diarios, y en ocasiones percibe un pobre apoyo social, por lo que es propenso a desarrollar alteraciones en su salud física, psicológica y social como consecuencia de su labor, mismas que se conocen como "síndrome de sobrecarga del cuidador", que, de no ser tratado oportunamente, puede acarrear enfermedades crónico-degenerativas y trastornos psicológicos. De igual modo, dicho síndrome puede ocasionar que la calidad de la atención que el cuidador provee al paciente se deteriore. Por ello, es necesario incluir a los cuidadores entre los receptores de atención psicológica (Maza, 2013; Valdez, 2009).

\section{Intervención psicológica en el paciente renal}

La evidencia señala que la intervención psicológica desempeña un papel relevante en la mejora de los problemas psicológicos asociados con las enfermedades médicas, así como en la calidad de vida del paciente y en su adherencia al tratamiento médico. En el área de la nefrología, la literatura señala la importancia de las variables psicológicas en el curso de la enfermedad; sin embargo, aún existen lagunas en el conocimiento derivadas de la escasez de estudios que examinen el papel de las intervenciones psicológicas en pacientes con ERC. Pascoe, Thompson, Castle, McEvedy y Ski (2017) realizaron una revisión sistemática con la finalidad de evaluar los efectos de las intervenciones psicosociales en la sintomatología ansiosa y depresiva, así como en la calidad de vida de los pacientes con ERC, hallando que dichas intervenciones tienen un efecto moderado en la reducción de la ansiedad y la depresión y en el incremento de la calidad de vida; no obstante, dicha revisión se llevó a cabo a partir de una cantidad reducida de estudios (ocho en total), lo que obliga a los referidos autores a sugerir el desarrollo de una mayor investigación que analice el papel de las intervenciones psicológicas en los pacientes con ERC, a fin de establecer con mayor certeza su efecto. 
Por lo anterior, es que se considera necesario reconocer la psiconefrología como una disciplina, como una rama de la psicología de la salud que utiliza los conocimientos y técnicas psicológicas en el estudio e intervención de los pacientes con ERC y de sus familias, así como del personal sanitario que los atiende, ocupándose de los aspectos emocionales, cognitivos y conductuales (Ortega, 2009).

Para favorecer la efectividad de sus tratamientos, la psiconefrología diseña intervenciones basadas en la evidencia, expresión que se refiere al hecho de fundamentar la praxis de la psicología en la mejor evidencia científica disponible, sustentándose en los siguientes pilares conceptuales: el fundamento empírico; las preferencias, valores y expectativas del paciente; el contexto, y la pericia del profesional para aplicar los tratamientos (Martínez, 2014).

Es recomendable que los programas de tratamiento en el campo de la psiconefrología tengan un corte semejante a la terapia breve pues, al tratarse de una enfermedad crónica, la intervención se efectúa regularmente en un ambiente hospitalario, donde la demanda de atención es cuantiosa, sobre todo en las instituciones públicas. Aunado a ello, en ocasiones la estancia intrahospitalaria de los pacientes es de corta duración, por lo que se dispone de poco tiempo para aplicar el tratamiento psicológico.

Las intervenciones no solo deben modificar y eliminar aquellas conductas y cogniciones desadaptadas, sino que también deben centrarse en la adquisición de las nuevas habilidades y comportamientos que habrán de incorporarse al repertorio conductual y cognitivo de los pacientes, que favorezcan la adaptación a la ERC y estimulen a las personas para que pongan en juego su capacidad para resolver conflictos. Al respecto, algunos autores consideran que el aprendizaje del optimismo favorece conductas más adaptativas de afrontamiento y, con ello, mejora la adherencia al tratamiento médico.

Con base en lo anterior, se ha podido identificar las principales actividades del especialista en psiconefrología, las cuales son las siguientes:

- Detectar necesidades de apoyo psicológico y de cambio conductual en pacientes y cuidadores mediante evaluaciones de su estado mental y emocional.
- Entrenar a los pacientes en estrategias que contribuyan a resolver los problemas psicológicos generados por los orgánicos.

- Favorecer alternativas de cambio en el estilo de vida para prevenir problemas o complicaciones orgánicas.

- Diseñar e instrumentar intervenciones que mejoren la adherencia a la terapéutica médica.

- Desarrollar habilidades en los cuidadores que les permitan soportar la sobrecarga.

- Entrenar a los familiares de los pacientes en estrategias de afrontamiento con la finalidad de que mejoren o mantengan su calidad de vida.

- Fomentar el desarrollo de habilidades lingüísticas e instrumentales para resolver problemas, así como mejorar la comunicación efectiva y la toma de decisiones razonadas entre los pacientes y sus cuidadores.

- Contribuir al desarrollo de habilidades de interacción y comunicación efectiva entre el personal sanitario involucrado y el paciente con ERC.

- Diseñar y poner en práctica programas de intervención para favorecer el bienestar psicológico en el personal de salud de los servicios de nefrología.

- Reportar en informes psicológicos y notas clínicas los resultados de las evaluaciones e intervenciones.

- Impulsar programas de formación profesional y difusión del trabajo realizado en el ámbito de las intervenciones psiconefrológicas en foros especializados y publicaciones periódicas.

- Construir instrumentos psicométricos específicos para personas con ERC.

En virtud de lo señalado, el psicólogo especialista en psiconefrología ejerce su función empezando con la evaluación conductual, seleccionando de entre una gama de estrategias aquellas que, además de contar con niveles apropiados de validez y confiabilidad, garanticen una adecuada operacionalización de las variables y se ajusten a las necesidades y características de la población a estudiar y al contexto donde se lleva a cabo la intervención. Algunas de las herramientas más utilizadas 
son las entrevistas, autorregistros, autoinformes, instrumentos psicométricos y medidas de evaluación psicofisiológica. Después, se identifican las respuestas conductuales, cognitivas y emocionales que se han de modificar, así como las variables que las originan y mantienen, y la interrelación entre las mismas, para luego seleccionar las técnicas que, de acuerdo con la evidencia, son las idóneas para conformar el tratamiento. La terapia cognitivo-conductual ha mostrado ser el modelo teórico con mayor eficacia en pacientes con enfermedades crónicas, pues identifica y modifica la causa que en ese momento está generando sufrimiento y previene su empeoramiento (Cabrales et al., 2017). Las técnicas derivadas de este enfoque favorecen la adaptación del paciente a la condición de salud y le ayudan a poner en práctica estrategias para su regulación emocional y mejorar su calidad de vida (Sánchez, Velazco, Rodríguez y Baranda, 2006).

Se describen a continuación las técnicas de intervención basadas en el enfoque cognitivo-conductual que más se utilizan en pacientes con ERC y en sus cuidadores primarios.

\section{Control de estímulos}

Se trata de una técnica dirigida a moderar o eliminar los estímulos que producen o fomentan la aparición y el mantenimiento de conductas que se desea suprimir o, en caso contrario, los que impiden o dificultan aquellas que se quiere implementar o aumentar. Para ello, el primer paso consiste en identificar y registrar los estímulos que producen o dificultan una conducta, según sea el caso, para luego seleccionar los que pueden manipularse según los objetivos terapéuticos. Con base en lo anterior, se planifica el ambiente del sujeto y finalmente se le proporcionan las instrucciones necesarias para modificar la conducta (Gavino, 2009).

En el servicio de nefrología, esta técnica se utiliza para lograr objetivos relacionados con la adherencia al tratamiento, como el apego al régimen alimenticio y la reducción de la ingesta de líquidos y el consumo de sal.

\section{Solución de problemas}

Es este un proceso cognitivo-conductual desarrollado por D'Zurilla y Goldfried (1971) que ayuda al paciente a disponer de una serie de alternativas de respuesta para afrontar situaciones problemáticas y aumentar así la probabilidad de elegir la solución más eficaz entre esas opciones. De acuerdo con Rodríguez y Gavino (2009), puede considerarse como un método de tratamiento que resalta la importancia de las operaciones cognitivas para comprender y resolver problemas, teniendo como ventajas el poder utilizarse para la resolución de conflictos y fomentar la autonomía, así como para mantener los resultados terapéuticos.

Los pasos para su aplicación son los siguientes: 1) Identificar y formular el problema y establecer objetivos; 2) Proponer diferentes alternativas de solución; 3) Evaluar las ventajas y desventajas de cada opción propuesta, para luego seleccionar la que se considere más adecuada; 4) Instrumentar la alternativa de solución elegida, y 5) Evaluar los resultados de esa solución.

En el servicio de nefrología, el objetivo de esta técnica radica en desarrollar en el paciente y en el cuidador primario las herramientas que les permitan generar por sí mismos las alternativas con las que cuentan para resolver los problemas y retos cotidianos que se presenten, así como para aprovechar los recursos cognitivos y conductuales de los que ya disponen.

Frecuentemente, debido a las características de la enfermedad, el rigor del tratamiento y el elevado requisito de respuesta implicado, los pacientes y cuidadores primarios perciben múltiples barreras para modificar su estilo de vida de manera óptima. A través de este procedimiento, se fomenta en ellos el desarrollo de habilidades instrumentales para producir y seleccionar opciones que les permitan enfrentarse de un modo realista y efectivo a las barreras percibidas. Su aplicación con pacientes con ERC resulta con frecuencia de utilidad en la toma de decisiones respecto a la modalidad del tratamiento de sustitución renal que elegirán, la mejora de la adherencia a la dieta, la ideación de alternativas para conseguir entradas adicionales de recursos económicos y la extensión de las redes de apoyo primarias y secundarias.

\section{Terapia cognitiva}

Desarrollada por Beck en 1962, opera bajo la hipótesis de que los pensamientos tienen efectos 
sobre las emociones y la conducta, por lo que la reestructuración de dichos pensamientos puede mejorar el estado de ánimo del paciente (Beck, 1976). Esta terapia sustenta el modelo llamado $\mathrm{ABC}$, que representa el ciclo de interacción entre pensamientos y conductas. Así, A son los estímulos o situaciones disparadoras; B los pensamientos automáticos, que no son resultado del razonamiento, que aparecen de manera espontánea, que se pueden presentar de forma visual o verbal y de los cuales surgen las creencias, mismas que son ideas que interpreta el sujeto como verdades absolutas, y que son las definiciones, evaluaciones o interpretaciones que hace de sí mismo, de los demás y del mundo; por último, la $\mathrm{C}$ se refiere a las consecuencias emocionales y conductuales.

La terapia cognitiva consiste en aprender a identificar los pensamientos irracionales que producen malestar emocional, para luego aprender a sustituirlos por ideas alternativas más realistas mediante un proceso de discusión y evaluación de los pensamientos negativos, cuestionando su veracidad, fomentando siempre un pensamiento crítico y apoyándose en hechos ajustados a la realidad (Ibáñez y Manzanera, 2011).

En los pacientes con ERC, es este un procedimiento utilizado con frecuencia. En el caso de las personas con reciente diagnóstico, con hospitalizaciones frecuentes, con trasplantes en los que hubo rechazo del injerto, por solo mencionar algunos ejemplos, se utiliza esta técnica para modificar pensamientos de poca valía, ideas de desesperanza, visión de túnel y pensamiento catastrófico acerca de su pronóstico y futuro. En los cuidadores primarios, es común encontrar pensamientos poco realistas acerca de la enfermedad y el tratamiento, los que usualmente son reforzados por algunos mitos populares. De igual modo, sobre todo en el caso de cuidadores con síndrome de sobrecarga, surgen ideas de autoculpabilización, pensamiento dicotómico, exigencias de perfeccionismo, despersonalización, ideas de poca valía e inutilidad, todo lo cual genera malestar emocional. Es necesario modificar estos pensamientos para promover la autorregulación emocional y aumentar la funcionalidad y las conductas de adherencia de pacientes y cuidadores.

\section{Técnicas de relajación}

Estas técnicas buscan producir una respuesta contraria a la activación fisiológica que se genera en situaciones de estrés a fin de facilitar la recuperación de la calma (Barrera, Fuentes y González-Forteza, 2013). Los cambios se encuentran mediados por el equilibrio del sistema nervioso autónomo en sus dos ramas: simpática, que regula la función de alerta y defensa, y la rama parasimpática, encargada de regular las funciones de ahorro y reposo. Mediante las técnicas de relajación se produce también un aumento en los niveles de serotonina, neurotransmisor que induce emociones como la alegría y las sensaciones de tranquilidad (Ruiz, Díaz y Villalobos, 2012).

Diversas investigaciones han documentado la eficacia de estas técnicas en el manejo del estrés, la ansiedad, el miedo, la depresión, la hipertensión arterial, el dolor y el insomnio (Johnson y Roberson, 2013; Linden y Moseley, 2006; Vázquez et al., 2009), aplicaciones que son congruentes con sus objetivos en los servicios de nefrología, donde además se suelen utilizar antes de llevar a cabo procedimientos médicos invasivos y quirúrgicos.

\section{Intervenciones psicoeducativas}

Consisten en programas estructurados en los cuales se provee información acerca de la enfermedad y el proceso de la misma, el tratamiento, los recursos y servicios de los que dispone, además de entrenar a los cuidadores para responder de manera adecuada a los desafíos relacionados con su labor y en el manejo de sus emociones. Este formato de intervención suele incluir materiales de lectura, discusiones en grupo y materiales impresos (Sörensen, Pinquart y Duberstein, 2002).

Las intervenciones psicoeducativas se emplean de manera recurrente en el área de la psiconefrología dado que frecuentemente los pacientes y sus familiares reciben el diagnóstico de forma inesperada y desconocen los términos relacionados con la ERC, motivo por el cual surgen en ellos múltiples dudas sobre el padecimiento y su tratamiento. Cabe mencionar que alrededor de la ERC y el tratamiento sustitutivo de la función renal hay múltiples mitos populares que producen una per- 
cepción equivocada acerca de los mismos, lo que conlleva el riesgo de que los pacientes y los cuidadores rechacen la propuesta de incorporarse a la diálisis o hemodiálisis, o bien tengan expectativas poco realistas acerca del trasplante renal. En virtud de lo anterior, este tipo de intervención suele ser útil para que conozcan la enfermedad y el tratamiento, desarrollen estrategias para afrontarlos y reduzcan el nivel de ansiedad que unos y otros manifiestan ante el diagnóstico y el tratamiento, ya que les da mayor sensación de seguridad y control en cuanto que logran saber más sobre ellos.

\section{Desafíos de la psiconefrología}

Uno de los desafíos más importantes a enfrentar es diseñar programas de calidad, y de intervención breve basados en la evidencia y que resulten adecuados para aplicarse en instituciones públicas en las que la demanda supera en gran medida al personal sanitario, donde los pacientes disponen de pocos recursos económicos para trasladarse en múltiples ocasiones al hospital, y no se cuenta con el espacio físico suficiente para cubrir las necesidades de atención.

Otro reto a superar es la inclusión de los profesionales de la psiconefrología que deciden formarse en esta disciplina, pues en la mayoría de los hospitales generales de México solo hay un psicólogo para atender las necesidades de todos los pacientes que acuden a recibir los servicios (Rivera, Díaz, Chapa, López y Zavala, 2015). Un desafío adicional es que los psicólogos que se integren al campo de la psiconefrología cuenten con el entrenamiento profesional necesario para la atención de esta población, que utilicen métodos de inter- vención basados en la evidencia, que conozcan a fondo la enfermedad desde una perspectiva biopsicosocial y que posean las aptitudes necesarias para trabajar de manera interdisciplinaria con el equipo de salud.

La psiconefrología requiere profesionales comprometidos que busquen promover cambios físicos, cognitivos, emocionales y conductuales en los pacientes con ERC. Esta actuación profesional especializada requiere el diseño y la inclusión de programas de formación. En México se han abierto espacios en diversos foros académicos, congresos, diplomados y programas de posgrado cuya meta es introducir al psicólogo en el trabajo con pacientes que padecen ERC, pero sin que se ofrezcan aún programas completos de entrenamiento en esta área, tal como existen ya en la de la psicooncología.

Para finalizar, es importante subrayar que uno de los objetivos primordiales de la psiconefrología es el de trabajar de manera interdisciplinaria para impulsar el bienestar del paciente con ERC y de su familia. Los equipos de salud incluyen idealmente al médico nefrólogo y a especialistas en enfermería, nutrición, trabajo social y psicología (María, 2003); sin embargo, en México, tal como apuntan Rivera et al. (2015), en las instituciones de salud todavía no se tienen las condiciones óptimas para desarrollar un trabajo verdaderamente interdisciplinario que incluya entre sus actores principales al psiconefrólogo. Por ello, se requiere de un esfuerzo común para generar espacios que permitan el entrenamiento y la intervención del psicólogo, y se favorezca así la diversificación de su labor con el propósito de brindar oportunamente un trato ético y digno al paciente con ERC.

\section{REFERENCIAS}

Aggarwal, H.K., Jain, D., Dabas, G. y Yadav, R.K. (2017). Prevalence of depression, anxiety and insomnia in chronic kidney disease patients and their co-relation with the demographic variables. Prilozi, 38(2), 35-44. doi: 10.1515/prilozi-2017-0020.

Amigo, I. (2014). Manual de psicología de la salud. Madrid: Pirámide.

Andrés, B. (2004). Diagnósticos de enfermería en pacientes con insuficiencia renal crónica en hemodiálisis. Revista de la Sociedad Española de Enfermería y Nefrología, 7(3), 158-163.

Ascencio, L. (2015). Psicooncología y cuidados paliativos. En J. A. Piña y J. Ybarra (Coords.): La psicología en el ámbito de la salud en México, investigación y aplicación del conocimiento (pp. 25-46). Ciudad Victoria, Tamps. (México): Universidad Autónoma de Tamaulipas.

Ávila S., M.N., Conchillos O., G., Rojas B., I.C., Ordoñez C., A.E. y Ramírez F., H.J. (2013). Enfermedad renal crónica: causa y prevalencia en la población del Hospital General La Perla. Medicina Interna de México, 29, 473-478. 
Barrera, V.M., Fuentes, I.P. y González-Forteza, C. (2013). Estrés y salud mental. Estrategias para el manejo integral del estrés. México: Instituto Nacional de Psiquiatría.

Bazán R., G. (2003). Contribuciones de la psicología de la salud en el ámbito hospitalario de México. Psicología y Ciencia Social, $5(1), 20-26$.

Beck, A.T. (1976). Cognitive therapy and the emotional disorders. New York: International Universities Press.

Cabrales A., J., Sánchez E., M., Ojeda V., I., Monterrubio F., E. y Hernández G., C. (2017). Factores asociados a ansiedad y desesperanza en pacientes con enfermedad renal crónica en un hospital regional de México. Revista Mexicana de Investigación en Psicología, 9(1), 46-53.

Calvanese, N., Feldman, L. y Weisinger, J. (2004). Estilos de afrontamiento y adaptación en pacientes sometidos a hemodiálisis. Revista de Nefrología Latinoamericana, 11, 49-63.

Conde, J.L. (1994). Aspectos económicos y organizativos del tratamiento de la insuficiencia renal crónica permanente. Nefrología, 14, 3-9.

Contreras, F., Esguerra, G., Espinoza, J.C., Gutiérrez, C. y Fajardo, L. (2006). Calidad de vida y adhesión al tratamiento en pacientes con insuficiencia renal crónica en tratamiento de hemodiálisis. Universidad Psicología, 5(3), 487-499.

Cvengros, J., Christensen, A. y Lawton, W. (2004). The role of perceived control and preference for control in adherence to a chronic medical regimen. Annals of Behavioral Medicine, 27(3), 155-161.

Ćwiek, A., Czok, M., Kurczab, B., Kramarczyk, K., Drzyzga, K. y Kucia, K. (2017). Association between depression and hemodialysis in patients with chronic kidney disease. Psychiatry Danub, 3, 499-503.

D’Zurilla, T.J. y Goldfried, M.R. (1971). Problem solving and behavior modification. Journal of Abnormal Psychology, 78, 107-126.

García, F., Fajardo, C., Guevara, R., González, V. y Hurtado, A. (2002). Mala adherencia a la dieta en hemodiálisis: el papel de los síntomas ansiosos y depresivos. Nefrología, 22(3), 2245-2252.

García, H. y Calvanese, N. (2008). Calidad de vida percibida, depresión y ansiedad en pacientes con tratamiento sustitutivo de la función renal. Psicología y Salud, 18(1), 5-15.

Gavino, A. (2009). Guía de técnicas de terapia de conducta. Madrid: Pirámide.

Hersh-Rifkin, M. y Stoner, M.H. (2005). Psychosocial aspects of dialysis therapy. En J. Kallenbach, C. Gutch, M. Stoner y A. Corea (Eds.): Review of hemodialysis for nurses and dialysis personnel. St. Louis, MO: Mosby Inc.

Ibáñez T., C. y Manzanera E., R. (2011). Técnicas cognitivo-conductuales de fácil aplicación en asistencia primaria (parte 2). Semergen, 40(1), 34-41. doi: http://dx.doi.org/10.1016/j.semerg.2011.07.020.

Iliescu, E.A., Yeates, K.E. y Holland, D.C. (2004). Quality of sleep in patients with chronic kidney disease. Nephrology, Dialysis \& Transplantation, 19, 95-99. doi: 10.1093/ndt/gfg423.

Johnson, D. y Roberson, A. (2013). The evaluation of the effectiveness of relaxation training and sleep hygiene education for insomina of depressed patients. Clinical Scholars Review, 6(1), 39-46. doi: https://doi.org/10.1891/1939-2095.6.1.39.

Lerma, A. (2012). Ensayo clínico controlado para el tratamiento de los síntomas de ansiedad y depresión en pacientes con insuficiencia renal crónica mediante una intervención cognitivo-conductual comparada con la atención médica convencional (Tesis doctoral inédita). México: Universidad Nacional Autónoma de México.

Liaveri, P., Dikeos, D., Ilias, I., Lygkoni, E., Boletis, I., Skalioti, C. y Paparrigopoulos, T. (2017). Quality of sleep in renal transplant recipients and patients on hemodialysis. Journal of Psychosomatic Research, 93, 96-101. doi: http://dx.doi. org/10.1016/j.jpsychores.2016.12.013.

Linden, W. y Moseley, J. (2006). The efficacy of behavioral treatments for hypertension. Applied Psychophysiology and Biofeedback, 31(1), 51-63. doi: 10.1007/s10484-006-9004-8.

María, E. (2003). Intervención psicológica en pacientes con insuficiencia renal crónica. En E. Remor, P. Arranz y S. Ulla (Orgs.): El psicólogo en el ámbito hospitalario (pp. 395-419). Bilbao: Desclée de Brouwer Biblioteca de Psicología.

Marques, M. y Barrientos, A. (2000), Insuficiencia renal crónica. En J. Arias, M. A. Aller, J. I. Arias e I. Aldamendi (Eds.): Enfermería médico-quirúrgica. Madrid: Tebar.

Martínez C., A., Navarro G., J.F. y Górriz T., J. (2012). Concepto y epidemiología de la enfermedad renal crónica en la diabetes mellitus. En J. Górriz, A. Martínez C., C. Mora F. y J. Navarro G. (Eds.): Diabetes y enfermedad renal crónica. Epidemiología, patogenia, factores de progresión y detección de la nefropatía diabética (pp. 3-6). Barcelona: Sociedad Española de Nefrología. Recuperado de http://www.elsevier.es/ficheros/nefro/otras_pubs/nefropatia_diabetica_mod_1.pdf.

Martínez T., A. (2014). Prácticas psicológicas basadas en la evidencia: beneficios y retos para Latinoamérica. Revista Costarricense de Psicología, 33(2), 63-78.

Maza, B. (2013). Realidad del cuidador informal. Una visión dirigida hacia el cuidador informal del enfermo en situación terminal. Tesis inédita de grado. Santander (España): Universidad de Cantabria.

Méndez D., A., Méndez B., J., Tapia Y., T., Muñoz, A. y Aguilar, L. (2010). Epidemiología de la insuficiencia renal crónica en México. Diálisis y Trasplante, 31(1), 7-11. doi: org/10.1016/S1886-2845(10)70004-7. 
Montero, X. (2013). Valoración integral y factores de cambio en la carga de cuidadores primarios informales de niños con cáncer. Tesis doctoral inédita. México: Universidad Nacional Autónoma de México.

Muñoz V., E., Vaca A., M., Romero, E., García, J., Bagdad, A. y Mahamed, N. (2010). Comportamiento sexual en los pacientes de hemodiálisis en Melilla. Revista de la Sociedad Española de Enfermería y Nefrología, 13(2), 113-118.

National Kidney Foundation (2002). K/DOQI Clinical practice guidelines for chronic kidney disease: Evaluation, classification and stratification. American Journal of Kidney Disease, 39, 1-266.

Oblitas, L. y Becoña, E. (Eds.) (2000). Psicología de la salud. México: Plaza y Valdés.

Organización Mundial de la Salud (2004). Adherencia a los tratamientos a largo plazo: Pruebas para la acción. Ginebra: OMS.

Ortega E., Z. (2009). La psiconefrología en el Perú. Temática Psicológica, 5(1), 27-33.

Páez, A., Jofré, M., Azpiroz, C., Bortoli, M. (2009). Ansiedad y depresión en pacientes con insuficiencia renal crónica en tratamiento de diálisis. Universitas Psychologica, 8(1), 117-124. Recuperado de http://www.redalyc.org/pdf/647/64712168010.pdf.

Pascoe, M., Thompson, D., Castle, D., McEvedy, S. y Ski, C. (2017). Psychosocial interventions for depressive and anxiety symptoms in individuals with chronic kidney disease: Systematic review and meta-analysis. Frontiers in Psychology, 8, 1-13. doi: 10.3389/fpsyg.2017.00992.

Pérez M., J., Llamas F., F. y Legido, A. (2005). Insuficiencia renal crónica: revisión y tratamiento conservador. Archivos de Medicina, 1(3), 1-10.

Piña, J. y Rivera, V. (2006). Psicología de la salud: algunas reflexiones críticas sobre su qué y su para qué. Universitas Psychologica, 5(3), 669-679.

Piña, J.A. e Ybarra, J. (2015). La psicología en el ámbito de la salud en México: investigación y aplicación del conocimiento. México: Universidad Autónoma de Tamaulipas.

Rivera L., A., Díaz D., M., Chapa A., C., López L., M.M. y Zavala, S. (2015). Cambios comportamentales que favorecen la calidad de vida de pacientes con enfermedad renal crónica. En S. Camacho y S. Galán (Comps.): Alternativas psicológicas de intervención en problemas de salud (pp. 93-110). México: El Manual Moderno.

Rivera L., A., Montero L., L.A. y Sandoval Á., R. (2012). Desajuste psicológico, calidad de vida y afrontamiento en pacientes diabéticos con insuficiencia renal crónica en diálisis peritoneal. Salud Mental, 35(4), 329-337.

Rodríguez N., C. y Gavino, A. (2009). Técnicas dirigidas al entrenamiento en solución de problemas y el control de las cogniciones. En A. Gavino (Ed.): Guía de técnicas de terapia de conducta (pp. 111-131). Madrid: Pirámide.

Rojas V., Y., Ruiz M., A.O. y Gónzalez S., R. (2017). Ansiedad y depresión sobre la adherencia terapéutica en pacientes con enfermedad renal. Revista de Psicología, 26(1), 1-13.

Ruiz M., A., Díaz M., I. y Villalobos, A. (2012). Manual de técnicas de intervención cognitivo-conductual. Bilbao: Desclée de Brower.

Sánchez R., S., Velasco F., L., Rodríguez C., R. y Baranda S., J. (2006). Eficacia de un programa cognitivo-conductual para la disminución de la ansiedad en pacientes médicamente enfermos. Revista de Investigación Clínica, 58(6), $540-546$.

Santillán, C. (2010). Diseño, implementación y evaluación de un programa de intervención educativa para pacientes con insuficiencia renal crónica. Revista Latinoamericana de Medicina Conductual. 1(1), 37-46.

Sörensen, S., Pinquart, M. y Duberstein, P. (2002). How effective are interventions with caregivers? A updated meta-analysis. The Gerontologist, 42(3), 356-372. doi: 10.1093/geront/42.3.356.

Valdez, J. (2009). Síndrome de sobrecarga en cuidadores primarios de pacientes con insuficiencia renal crónica en diálisis peritoneal del Hospital General de Zona Número 8 IMSS “Gilberto Flores Izquierdo” en el periodo marzo, $2008-f e b r e r o, 2009$. Tesis inédita de especialidad. México: Universidad Nacional Autónoma de México.

Vázquez M., I., Vital F., S., Bravo G., M., Jurado C., S. y Reynoso E., L. (2009). Retroalimentación biológica y relajación en pacientes con enfermedad renal crónica terminal en tratamiento de hemodiálisis. Journal of Behavior, Health \& Social Issues, 1(2), 35-43. doi: http://dx.doi.org/10.5460/jbhsi.v1.2.18801.

Weiner, H. (1982). Psychobiological factors in bodily desease. En T. Millon, C. Green y R. Meagine (Eds.): Handbook of clinical health psychology (pp. 31-53). New York: Plenum Press.

World Health Organization (1946). Frequently asked questions. Geneve: WHO. Recuperado de http://www.who.int/suggestions/ faq/en/. 